\title{
FACTORESQUE AFECTAN LA PRODUCCIÓN DE LECHE EN VACAS DE DOBLE PROPÓSITO EN TRÓPICO HÚMEDO (PUCALLPA)
}

\author{
Sonia Sheen R. ${ }^{1}$ y Alfredo Riesco D. ${ }^{2}$
}

\section{ABSTRACT}

The effect of feeding, crossbreeding level, lactation period, and reproductivestatus on milk yield was eval uated in dual purpose cows in the humid tropics of Peru. A total of 204 cows from 23 farms were monitored. All cows grazed over Brachiaria decumbens. Energy supplementation consisted of brewery residues and ricebran. Cows weregrouped according to thelevel of European blood in Low, Medium, and High. Regression analysis and the " $t$ " Student test wereappl ied for statistical comparisons. Thehigher avai lability of B. decumbens had a negative effecton milk yield. Supplemented ani mals syielded moremilk $(5.2 \mathrm{~kg} / \mathrm{cow} / \mathrm{day})$ than those non-supplemented $(3.8 \mathrm{~kg} / \mathrm{cow} /$ day). Thelevel of European inheritanceaffected milk yield, where $50 \%$ crossbred cows had the highest milk yield ( 5.0 $\mathrm{kg} / \mathrm{cow} / \mathrm{day}$ ). Month of lactation affected productive performance Non-pregnant cows produced more milk than the pregnant ones ( $4.5 \mathrm{vs} .3 .7 \mathrm{~kg} / \mathrm{cow} /$ day). It is concluded that milk yield was affected by all variabl es under eval uation, especially thelevel of European blood.

K ey words: cows, milk yield, crossbred, feeding, lactation, reproduction, tropics

\section{RESUMEN}

Se evaluó el efecto de la alimentación, grupo racial, etapa de lactancia y estado reproductivo sobre la producción láctea en vacas de doble propósito en trópico húmedo. Se utilizaron 402 vacas distribuidas en 23 fundos. Todas las vacas pastoreaban sobre Brachiaria decumbens. La suplementación energética consistió en residuo de cervecería y/o polvillo dearroz. Las vacas fueronasignadosa 3 grupos raciales (Bajo, Medio y Alto mestizajeeuropeo). El análisis estadístico fue por regresiones y " $\mathrm{t}$ " Student. Los resul tados indicaron que el promedio de producción láctea fue de $4.3 \mathrm{~kg} / \mathrm{vaca} / \mathrm{dí}$. La mayor disponibilidad de B. decumbens tuvo un efecto negativo sobre la producción de leche. A ni mal es suplementados produjeron más leche $(5.2 \mathrm{~kg} / \mathrm{vaca} / \mathrm{día}$ ) que los no suplementados (3.8 kg/vaca/día). El grupo racial afectó la producción de leche, dondelas vacas con medio mestizaj eeuropeo fueron los quemás lecheprodujeron ( $5.0 \mathrm{~kg} / \mathrm{vaca} / \mathrm{día})$. El mes de lactancia afectó $\mathrm{e}$ rendimiento lácteo de los animales, y las vacas vacías produjeron más lecheque las gestantes ( $4.5 \mathrm{vs}$. $3.7 \mathrm{~kg} / \mathrm{vaca} / \mathrm{día}$ ). Se concluyeque la producción de leche estuvo afectada por todas las variables en estudio, pero principal mente por el tipo racial.

Palabras clave: vacas, producción de leche, grupo racial, alimentación, lactancia, reproducción, trópico

\footnotetext{
${ }^{1}$ Facultad de Zootecnia, Universidad Nacional Agraria de la Selva, Tingo María

${ }^{2}$ Consorcio para el Desarrollo Sostenible de Ucayali, CODESU
} 


\section{INTRODUCCIÓN}

La cría del ganado de doble propósito con fines de producción de leche se ha venido intensificando durantelaúltimadécadaen laRegiónUcayali (selvabajade Perú). Este sistema de explotación secaracteriza por tener vacas al pastoreo con amamantamiento de sus terneros, un ordeño al día, escaso uso desuplementosal imenticios, y diversidad de grupos raciales. Este sistema de producción es similar a los existentes en muchas regiones del trópi co húmedo latinoamericano.

El promedio de producción delechede los animales de la región es bastante bajo ( 3 kg/vaca/día, Riesco etal., 1985). Algunos de Iosfactoresquecondicionanestenivel deproductividad pueden ser cuantificados, y sepodrían generar al ternativas desolución queme jorarían el nivel deingreso alos productores. El presente trabajo sellevó a cabo con la final idad de eval uar el efecto de la alimenta ción, grupo racial, etapa de lactancia y estado reproductivo sobrela producción deleche en vacas de doble propósito del trópico húmedo de Pucallpa.

\section{Materiales y Métodos}

Lugar de Estudio

El trabajo se realizó en la zona de Pucal lpa, Ucayali, Penú, comprendiendo como área deestudio los fundos ganaderos productores deleche. Laépoca deestudio comprendiólos meses demayor precipitación pluvial (octubre-marzo).

Ucayali se encuentra ubicada en la partecentral y oriental del territorio peruano. Sus coordenadas geográficas se sitúan entre los 7029' 23" y 11027' 35" delatitud sur y los 70029' 46" y 750 58' 08" longitud oeste, y a una altura de 160 a 270 ms.n.m El prome dio deprecipitación pluvial anual es de1,700 $\mathrm{mm}$, con unatemperaturamediaanual de27.6 口C y humedad relativa de $83.6 \%$. La zona corresponde al ecosistema de bosque tropical semi siempre verde.

\section{A nimales}

Los animales en estudio fueron 402 vacas con ternero al pie distribuidos en 23 fundos de características similares con rela ción a instalaciones para el ordeño y pastoreo del ganado.

\section{Producción de leche}

La producción de leche se registró como el peso de la leche del bal de (en $\mathrm{kg}$ ) y la leche consumida por el ternero (dado por la diferencia de peso del ternero antes y después del amamantamiento). El ordeño fue manual y con apoyo del ternero.

Grupo racial

La clasificación de los animales fue por grupo racial, teniendo como referencia a Vaccaro (1993). Los grupos raciales fueron:

a) Bajo mestizajeeuropeo. Animalesconalto grado de sangreNelloreo Brahman), ( $n=$ 111 vacas).

b) Mediomestizajeeuropeo. Animalesnacidos porcnucecebuino(NelloreoBrahman) con razas europeess (Holstein o B. Swiss), a través de inseminación artificial o monta natural ( $n=212$ vacas).

c) Alto mestizajeeuropeo. Animalestraídos del exterior del país y de la sierra del Perú, con alto porcentaje de sangre europea ( $n=79$ vacas).

Etapa de lactancia

Se clasificó a los animales según el mes de lactancia postparto.

Estado reproductivo

Setuvo 2 grupos: preñadas de $1 \mathrm{a} 5 \mathrm{me}$ sesdegestacióny vacias. El estado reproductivo se determinó por pal pación rectal . 


\section{Alimentación}

La toma de datos comprendió la cla sificación botánica, disponi bilidad dela pastura y el consumo de insumos suplementarios. La pastura seeval uó siguiendo el método modificado de Haydock y Shamn (Senra y Venereo, 1979) o rendimiento comparativo. El consumo de insumos suplementarios se eval uó duranteel ordeño, siendo estos polvillo de arroz y/o residuo de cervecería La carga animal fuedadapor e número deunidadesanimal por hectárea, considerando la unidad animal igual a una vaca de $400 \mathrm{~kg}$ depeso vivo.

\section{A nálisis estadístico}

La respuesta de la producción de le chepor efecto de las variables en estudio fue analizada mediante el análisis de regresión lineal y cuadrática múltiple. Lasignificancia estadística de los coeficientes en las regresiones se midió a través de la prueba de "t" Student

\section{RESULTADOS Y DISCUSIÓN}

Disponibilidad de Brachiaria y producción de leche

El efecto de la disponibilidad de Brachiaria en la producción láctea medido através del coeficientederegresión muestra un efecto negativo ( $p>0.05)$ como seaprecia en la Fig.1. La alimentación es uno de los principales factores que afectan la producción deleche(Mol y Tyrrel, 1995) y seespera que la abundancia de pastura tenga un efecto positivo; sinembargo la respuestaproductiva del bovino al pastoreo es bastante compleja (Riesco y Seré, 1984; Vélez, 1997; UNAM, 1984; Ormazabal y Osoro, 1995).

En promedio, el conjunto delosfundos en estudio no adolecen de disponi bilidad de pastura, sino que es muy probable que viene siendo sub utilizada, tanto en cantidad (cargas animales bajas) como en cal idad. Exceso de pasto por unidad ani mal se ha reporta do con anterioridad en la zona de Pucallpa (UNMSM-IVITA, 1989; CIAT, 1998). EI Brachiaria un pasto ampliamente aceptado por los ganaderos (Riesco et al., 1985); y se recomienda una modal idad deuso intensivo, a una edad entre 3 a 4 semanas, debido a su mayor conteni do nutricional, mejor consumo y mayor productividad animal (Echevarría, 1994).

La disponibilidad de materia seca no necesariamente implica que habrá una mayor producción deleche, como seobservaen los resultados obteni dos. Cuando e forraje está muy crecido con hojas dispersas en diversos niveles y direcciones, la ingestión de materia seca por bocado es menor (Velez, 1997). Además, el val or nutritivo del forraje disminuye conforme avanza su madurez. Al disminuir la calidad del forraje ofrecido disminuyeladigestibilidad (Rearte, 1992; Otoya, 1984) y aumenta el conteni do de fibra cruda (Echevaría, 1994; Garcia, 1992), repercutiendo negativamente sobre la di sponi bilidad de proténay energía, y por lo tanto afectando la producción de leche Con cargas animales mayores de 2 U.A./ha en Brachiaria se puede conseguir producciones que van de 6 a 12.4 It/vaca/día (UNMSM-IVITA, 1989; Sánchez, 1988).

Suplementación y producción de leche

La producción de leche medida a través delasuplementación con polvillo dearroz y/o residuo de cervecería que recibían las vacas al pastoreo, indica un efecto positivo ( $>0.05$ ). El 38.5\% recibían suplementoy pre sentaron una producción promedio de $5.2 \mathrm{~kg} /$ vaca/día, en tanto queel grupo queno recibía tuvo una de $3.8 \mathrm{~kg} / \mathrm{vaca} / \mathrm{día}$

La al imentación suplementaria es una alternativa que el productor utiliza (CATIE, 1984), y quepermitemejorar los niveles productivos (Rearte, 1992). El residuo decervecería se utiliza en la zona de Pucal lpa con excelentes resultados (De la Torre, 1986; CIAT, 1998), y que permiteal canzar producciones de 8.4 kg/vaca/día (Sánchez, 1981). 


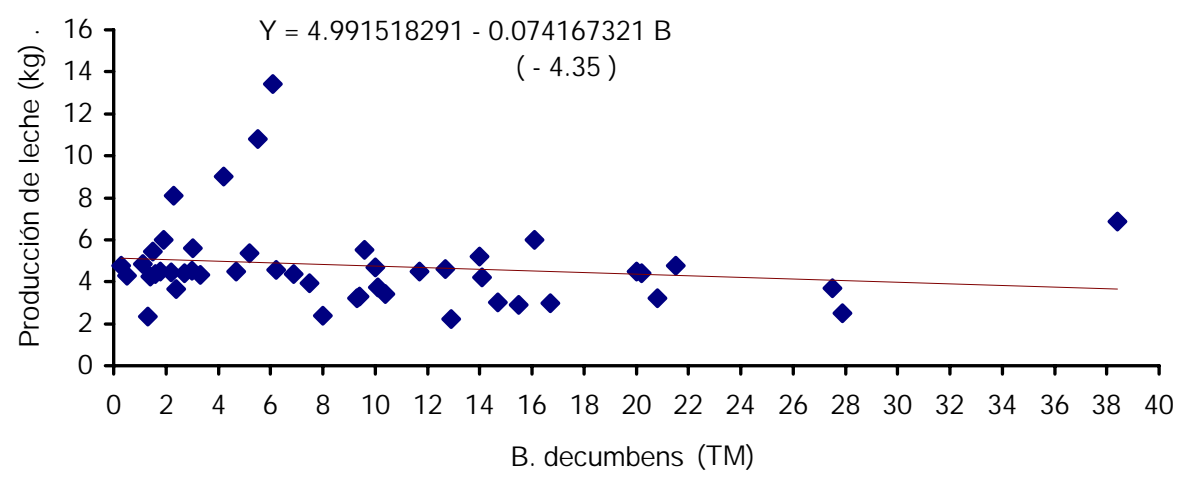

Figura 1. Disponibilidad de B. decumbens (materia seca) y producción de leche

Lasensı DıIIdaa de respuesta al supıe mento en vacas al pastoreo en trópico aún no está definida I nformaciones provenientes de climastempladosindican quecon un concentrado bal anceado se puede producir $2 \mathrm{~kg}$ de leche por kg desuplemento (Velez, 1997).

Lafaltadedisponibilidad desuplementos y el costo son causal es importantes para que el productor tenga un bajo índice de insumos en la alimentación de animales al pastoreo, tal como se encontró en el presente estudio. Lo más rentable pudiera ser para el productor usar poco o nada de concentrado (Campos y Wilcox , 1994). En todo caso, la metodología mas viablees el manejo adecuado de la pastura (Rearte, 1992).

Grupo racial y producción de leche

Lainfluenciadel grupo racial enlaproducción láctea se muestra en la Fig. 2. El mejor comportamiento productivo lo tuvo el medi o mestizajeeuropeo (MME) con un rendimiento de $5.0 \mathrm{~kg} / \mathrm{vaca} / \mathrm{d}$ ía Estetipo decomportamiento productivo también es reportado por Vaccaro (1993). El grupo de al to mestizaje europeo (AME) presentó la menor producción deleche $(2.9 \mathrm{~kg})$ y sin mayores ventajas frenteal os otros dos grupos; resultados que coinciden con Villegas y Román (1986) y Vaccaro (1993).

La ecuación de regresión cuadrática es la que mejor describeel comportamiento deıs anı mai es. tsteetecto cuaaran co positivo ( $p>0.05$ ) sehace manifiesto conformed grupo racial aumenta hasta media sangre. Si el grado sanguíneo europeo es mayor, este efecto se hace negativo. Se deduce que los comportamientos productivos entregrupos se pueden colocar en el siguiente orden: MME $>$ BME > AME.

Estado reproductivo y producción de leche

El $23.1 \%$ de las vacas evaluadas se encontraban preñadas, con gestaciones de 1 a 5 meses, y con una producción promedio de $3.7 \mathrm{~kg} / \mathrm{dí}$ a de leche, en comparación con la producción de las vacas vacías que fue de $4.5 \mathrm{~kg} / \mathrm{dí}$. Este efecto negativo ( $p>0.05$ ) de la preñez, es un aspecto importante del cual se tiene poca información (Broster y Swan, 1992) .

Seconocequelas hembras gestantes deben recibir mayores aportes de energía y otros nutrientes para permitir el desarrollo del feto, crecimiento uterino, producción y mantenimiento (Bondi, 1989), pero también se señal a que los primeros cinco meses de gestación tienen poco efecto sobre la lactancia (Broster y Swan, 1992). Los resultados encontrados inducen a pensar queen el trópico, bajo un sistema de al imentación fluctuante en calidad, las vacas gestantes estańan priorizando direccionar nutrientes al desarrollo del feto en detrimento dela producción deleche. 


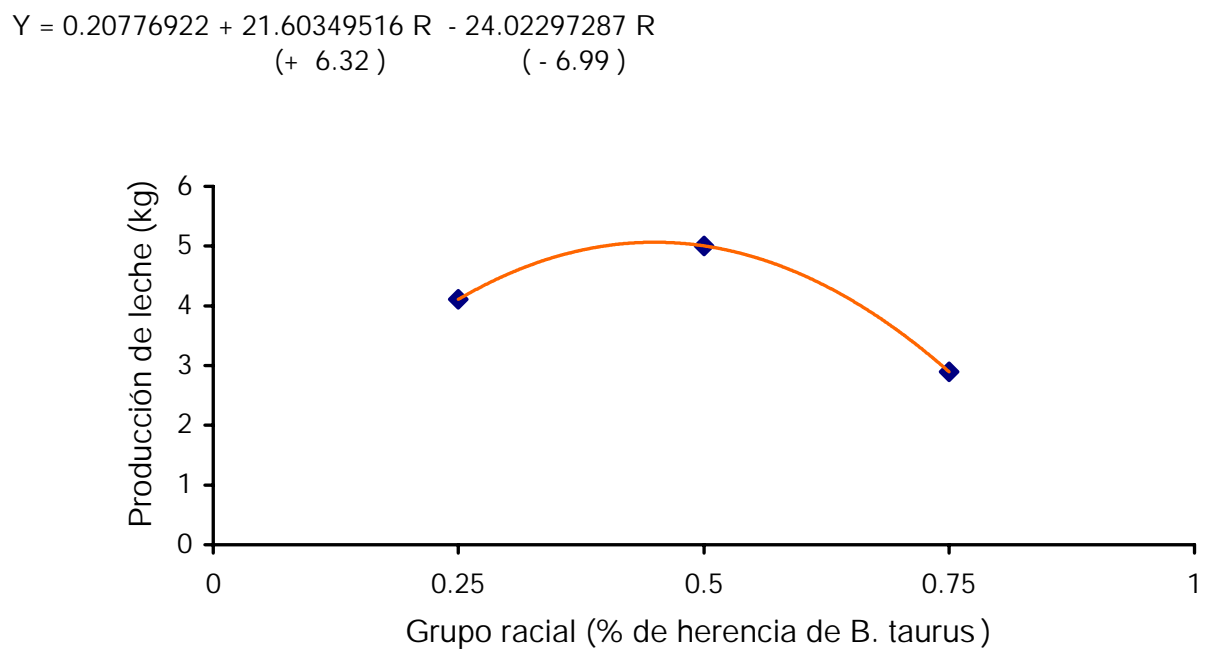

Figura 2. Grupo racial y producción de leche

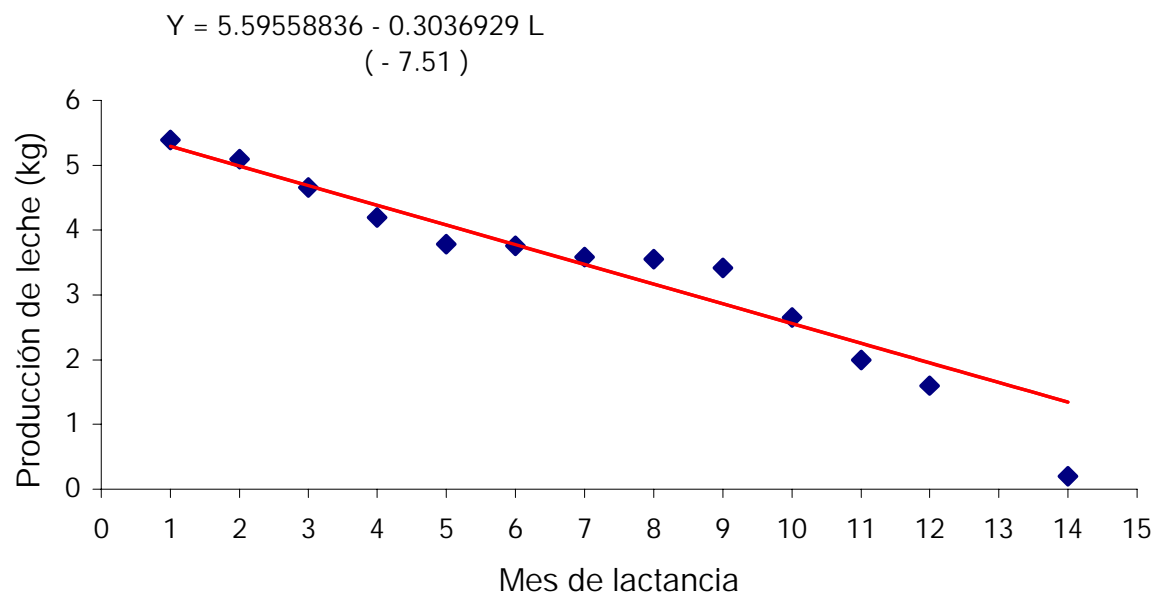

Figura 3. Etapa de lactancia y producción de leche

Etapa de lactancia y producción de leche

El comportamiento productivo por efecto de esta variable se aprecia en la Fig. 3. La curva de lactancia es descendiente, dondelas mayores producciones ocurren entrelas dos a seis semanas post parto (Broster y Swan, 1992; Harvey y Hill, 1969), seguido deun descenso paulatino. El mayor nivel de producción $(5.4 \mathrm{~kg})$ de leche se observa en el primer mes de lactancia, y desciende hasta $0.2 \mathrm{~kg}$ en vacas con 14 meses de paridas.
Se considera que en los 100 primeros días de lactancia la vaca produce al rededor del 40\% desu producción total (Velez, 1997).

Expresión de las variables independientes y producción de leche

Los efectos de las variables independientes en la producción deleche, medidosa través de la regresión múltiple, se describen en el Cuadro 1. El modelo expresa que el $26.7 \%$ de la variación de la producción de 
Cuadro 1. Efecto de las variables independientes sobre la variable dependiente (producción deleche)

\begin{tabular}{lccc}
\hline \multicolumn{1}{c}{ Descripción } & $\begin{array}{c}\text { Coeficientede } \\
\text { regresión }\end{array}$ & Error estándar & "t" \\
\hline Intercepto & +2.75568837 & - & - \\
Efecto de grupo racial $(\mathrm{R})$ & +13.18157934 & 3.5757 & $+3.69 * *$ \\
Efecto del grupo racial $\left(\mathrm{R}^{2}\right)$ & -14.22486723 & 3.7146 & $-3.83 * *$ \\
Efecto de etapa de lactancia (L) & -0.25977184 & 0.0416 & $-6.23 \mathrm{NS}$ \\
Efecto de estado reproductivo (E) & -0.38269875 & 0.2634 & $-1.45 \mathrm{NS}$ \\
Efecto deBrachiaria (B) & -0.02979251 & 0.0171 & $-1.73 \mathrm{NS}$ \\
Efecto de suplementación (S) & +0.97277089 & 0.2245 & $+4.33 \mathrm{NS}$ \\
\hline
\end{tabular}

leche está en función de las variables grupo racial $(R)$, etapa de lactancia $(L)$, estado reproductivo(E), disponibilidad deBrachiaria (B) y suplementación energética (S), con un coeficiente de variación de $45.3 \%$. El comportamiento delas variables essimilar acuando seanal izan en forma independiente.

\section{Literatura Citada}

1. Bondi, A. 1989. Nutrición Animal. Ed. Acribia, S.A. Zaragoza, España. p 421428.

2. Broster, W.; T. Swan. 1992. Estrate gias de alimentación para vacas leche ras deal ta producción. AGT Editor, S.A. México. 380 p.

3. Campos, M.; Ch. Wilcox. 1994. Estra tegias de al imentación y sistemas de producción de leche para América Latina. Universidad de Florida. Gainesville. EEUU. p 93-98.

4. Centro Agronómico Tropical de Investigación y Enseñanza. 1984. Compila ción de documentos presentados en actividades decapacitación. Turrial ba, Costa Rica. 121 p.

5. Centro de Investigación de Agricultura Tropical (Cl AT).1998. Ex ante analysis of new forage alternatives in farms with dual purpose cattle in Peru, Costa Rica and Nicaragua. CIAT, Colombia p 35-46.

6. Dela Torre, M. 1986. Comparación de dos sistemas de producción de leche al pastoreo en la Amazonía. IX Reunión Cientúfica deProducción Animal.

7. Echevarría, M. 1994. Alimentación del ganado con pastostropicales. Departamento deNutriciónAnimal, UniversidadNacional Agraria (UNA), Lima Perú. p 2-27.

8. García, G.F. 1992. Requerimientos de proteŕna en ganado lechero. Simulación de sistemas pecuarios. RISPAL. IICA. Costa Rica. p 105-131.

9. Harvey, C.; H. Hill. 1969. Leche, producción y control. Ed. Academia. Barcelona, España. p 591.

10. Mol, P.W; H.F. Tyrrell. 1975. Efficiency of conversion of digestibleenergy to milk. J. Dairy Sci. 58: 602 - 617.

11. Ormazabal, J .; K. Osoro. 1995. Efecto del pasto disponi bleen la producción y calidad de la leche y en la ganancia de terneros. VI J ornadas sobre Producción Animal. ITEA. № 16 , Tomo I. España. p195-197.

12. Otoya, Z.V. 1984. Calidad nutritiva del pasto Braquiaria (Brachiaria decumbens) y su respuesta en la producción de leche. Tesis Bach., Fac. Medicina Veterinaria, 
Universidad Nacional Mayor de San Marcos. Lima, Perú. 47 p.

13. Rearte, D. 1992. Alimentación y composición de la lecheen los sistemas pastoriles. EEA. CERBAS. INTA. Centro Regional Buenos Aires Sur. Argentina. $\mathrm{p} 94$.

14. Riesco, A.; M. De La Torre; C. Reyes; G. Meini; H. Huamán; M. García. 1985. Análisis exploratorio delos sistemes de fundos de pequeños productores en la amazonía. Pucallpa Perú. $47 \mathrm{p}$.

15. Riesco, A.; C. Seré. 1984. Análisis económico de resultados de las pruebas de pastoreo. Evaluación de pasturas con animales, al ternativas metodológicas CIAT-RIEPT. Colombia. p 201-231.

16. Sánchez, M.J . 1981. Utilización de diferentesniveles deresiduo decervecería en raciones de vacas en producción. Tesis Ing. Zoot, Fac. Zoot, UNAS. Tingo María $52 \mathrm{p}$.

17. Sánchez, V.M. 1988. Diagnóstico estático de los sistemas de producción agraria de la zona de Soritor. Tesis Ing. Zoot, Universidad Nacional Agraria de laSelva, Tingo María, Perú. 69 p.

18. Senra, A.; A. Venereo. 1979. Métodos de muestreo. Los pastos en Cuba. Tomo
I. Instituto deCienciaAnima. LaHabana p449-490.

19. Universidad Nacional Autónoma de México (UNAM). 1984. Centro de Investigación, Enseñanzay Extensiónen Ganadería Tropical. Facultad deMedicina Veterinariay Zootecnia. Boletín. 230 p.

20. Universidad Nacional Mayor de San Marcos (UNMSM), IVITA-CIID. 1989. Sistemas de Producción Amazónicos. Segundo informe (1984-1986). Pucallpa. Perú. $79 \mathrm{p}$.

21. Vaccaro, L. 1993. Caracterización de 18 rebaños venezolanos dedoblepropósito. Facultad de Agronomía y de Ciencias Veterinarias, Universidad Central deVenezuela. XII Reunión Latinoamericanade Producción Animal, ALPA. Santiago de Chile

22. Velez, M. 1997. Producción de ganado lechero en el trópico. Ed. Línea Gráfica. Escuela Agrícola Panamericana, Zaruaraus. Honduras. $183 \mathrm{p}$.

23. Villegas, M.; P.H. Román. 1986. Producción de leche durante el proceso de formación de un rancho de doble propósito en el trópico. Técnica Pecuaria №51. México. p 51-61. 